\title{
Target of selective auditory attention can be robustly followed with MEG
}

\author{
Dovilè Kurmanavičiūtè ${ }^{\mathrm{a}, *}$, Antti Rantala ${ }^{\mathrm{a}}$, Mainak Jas ${ }^{\mathrm{a}, \mathrm{c}}$, Anne Välilä ${ }^{\mathrm{a}}$, \\ Lauri Parkkonen ${ }^{\mathrm{a}, \mathrm{b}}$ \\ ${ }^{a}$ Department of Neuroscience and Biomedical Engineering, Aalto University, P.O. Box \\ 12200, FI-00076 Aalto, Finland \\ ${ }^{b}$ Aalto NeuroImaging, Aalto University, FI-00076 Aalto, Finland \\ ${ }^{c}$ CNRS LTCI, Télécom Paris Tech, Université Paris-Saclay, Paris, France
}

\begin{abstract}
Selective auditory attention enables filtering relevant from irrelevant acoustic information. Specific auditory responses, measurable by electro- and magnetoencephalography (EEG/MEG), are known to be modulated by attention to the evoking stimuli. However, these attention effects are typically demonstrated in averaged responses and their robustness in single trials is not studied extensively.

We applied decoding algorithms to MEG to investigate how well the target of auditory attention could be determined from single responses and which spatial and temporal aspects of the responses carry most of the information regarding the target of attention. To this end, we recorded brain responses of 15 healthy subjects with MEG when they selectively attended to one of the simultaneously presented auditory streams of words "Yes" and "No". A support vector machine was trained on the MEG data both at the sensor and source level to predict at every trial which stream was attended.

Sensor-level decoding of the attended stream using the entire 2-s epoch resulted in a mean accuracy of $93 \% \pm 1 \%$ (range $83-99 \%$ across subjects). Time-resolved decoding revealed that the highest accuracies were obtained 200-350 ms after the stimulus onset. Spatially-resolved source-level decoding indicated that the cortical sources most informative of the attended stream were located primarily in the auditory cortex, especially in the right hemisphere.
\end{abstract}

\footnotetext{
*Corresponding author dovile.kurmanaviciute@aalto.fi
} 
Our result corroborates attentional modulation of auditory evoked responses also to naturalistic stimuli. The achieved high decoding accuracy could enable the use of our experimental paradigm and classification method in a brain-computer interface.

Keywords: selective attention, single-trial analysis, magnetoencephalography, MEG, auditory system, dichotic listening 


\section{Introduction}

Selective auditory attention is a cognitive function which enables filtering of relevant information from irrelevant. The need of such a selection mechanism has been illustrated by the cocktail party problem in which the listener has to concentrate his/her auditory attention to one speaker while suppressing the voices of the irrelevant speakers to follow that one speaker (Cherry, 1953). Electroencephalographic measurements during dichotic listening have shown that selective auditory attention modulates brain responses generated in auditory cortex (Hillyard et al., 1973; Woldorff et al., 1993).

In the last decade, machine-learning methods have been applied to test whether the target of selective attention can be detected from electro- and magnetoencephalographic (EEG/MEG) data (Nijboer et al., 2008; Furdea et al., 2009; Halder et al., 2010, 2016; Schreuder et al., 2010; Höhne et al., 2011; Hill et al., 2012; Nambu et al., 2013; Hübner et al., 2018). EEG and MEG are well suited for monitoring attention effects as they provide a high temporal resolution in the order of milliseconds, enabling the detection and classification of evoked responses (e.g. auditory or visual P300; McCane et al., 2015; Yeom et al., 2014; Curtin et al., 2012), steady-state responses (e.g. SSRs or mixed SSR/P300; Kaongoen and Jo, 2017; Kim et al., 2011) and oscillatory brain activity (e.g. sensory-motor rhythm (SMR); Geronimo et al.).

The ability to detect the target of auditory attention from brain signals has been exploited to improve the performance of hearing aids (Kidd, 2017) as well as in brain-computer interfaces (BCI) e.g. to re-enable communication in paralyzed patients (Sellers and Donchin, 2006; Astrand et al., 2014; McCane et al., 2015). However, attentional effects are not equally easy to detect from all response types. Hill and colleagues (2012) argue that attention-based classification on ERPs is more reliable than that on steady-state evoked potentials (SSEPs) in a dichotic listening task due to the limited attentional modulation of auditory SSEPs.

Many BCI approaches employ a secondary mental task artificially connected to the primary task because of the poor signal-to-noise ratio of the responses related to it; for example, a primary task of communicating a "yes" or "no" answer could be linked to a secondary task of imagining moving the right or left hand, respectively. Here, we will test the use of spoken-word stimuli in BCI that only comprises the primary task and thus requires minimal training of the subjects. 


\section{Materials and methods}

\subsection{Participants}

Fifteen healthy adult volunteers ( 4 females, 11 males; mean age $28.8 \pm 3.8$ years, range $23-38$ years) participated in our study. Two subjects were lefthanded and the rest right-handed. Participants did not report hearing problems or history of psychiatric disorders. The study was approved by the Aalto University Ethics Committee. All participants gave their informed consent prior to the recordings.

First Block "Attended Left"

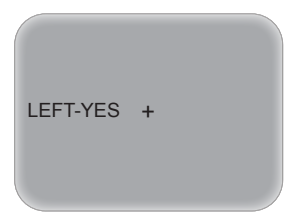

$\sim 135 \mathrm{~s}$
Second Block "Attended Right"
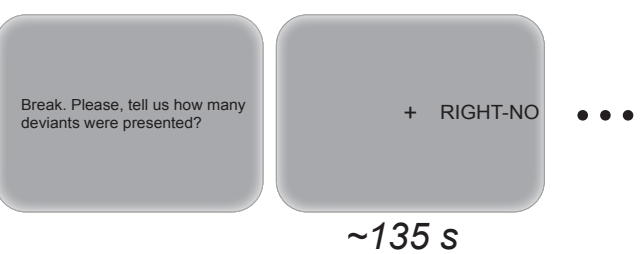

Time

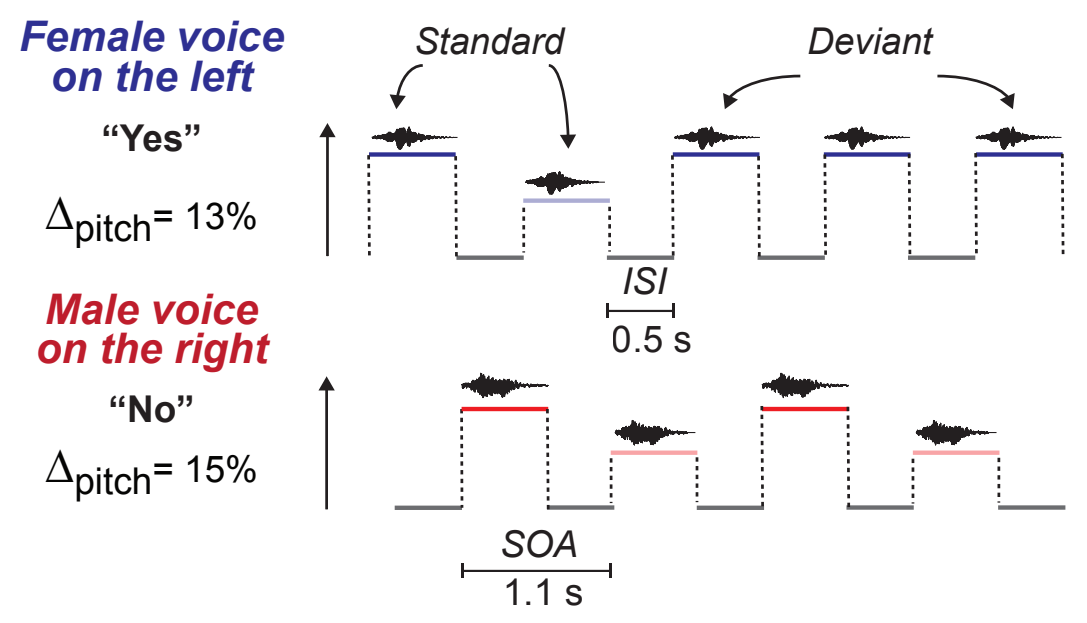

Figure 1: Experimental design. Top: Block structure and the instructions to the subject. Bottom: Stimulus sequence within each block. 


\subsection{Stimuli and experimental protocol}

The auditory stimulus comprised of two simultaneous word streams; the word "Yes" was repeatedly presented on the left side and the word "No" on the right; see Figure 1. In each word stream, high- and low-pitch versions of the same word stimuli alternated. To control for subjects' attention, the sequence contained occasional deviants (violations of the regular alternation), which comprised three consecutive high-pitch versions of the same word. Deviant probability was $10 \%$ in both streams for the first seven subjects and $5 \%$ for the rest subjects in order to reduce the mental load of memorizing the deviant count.

To create a realistic acoustic scene, the stimuli were recorded with a dummy head at the center of a room with dimensions comparable to those of the magnetically shielded room where the MEG recordings were performed. The speakers were standing at about 40 degrees to the left/right of the dummy head at a distance of $1.13 \mathrm{~m}$.

The experiment comprised 8 blocks, each lasting about 5 min. Two seconds before a block started, the subject was instructed to direct his/her attention to one of the streams by the cues "LEFT-YES" or "RIGHT-NO" on the screen. The task of the subject was to focus on the indicated word stream, covertly count the deviants and maintain gaze at the fixation cross displayed on the screen. The experiment always started with the condition "Attended Left" and was followed by the condition "Attended Right". The order of the remaining six blocks was randomized across subjects. The total length of the experiment was 50-60 minutes including the breaks between the blocks.

PsychoPy version 1.79.01 (Peirce, 2007, 2008) Python package was used for controlling and presenting the auditory stimuli and visual instructions. The stimulation was controlled by a computer running Windows 2003 for the first nine subjects and Linux Ubuntu 14.04 for the rest. Auditory stimuli were delivered by a professional audio card (E-MU 1616m PCIe, E-MU Systems, Scotts Valley, CA, USA), an audio power amplifier (LTO MACRO 830, Sekaku Electron Industry Co., Ltd, Taichung, Taiwan), and custombuilt loudspeaker units outside of the shielded room and plastic tubes conveying the stimuli separately to the ears. Sound pressure was adjusted to a comfortable level for each subject individually. 


\subsection{MEG data acquisition}

MEG measurements were performed with a whole-scalp 306-channel Elekta Neuromag VectorView MEG system (Elekta Oy/MEGIN, Helsinki, Finland) at the MEG Core of Aalto Neuroimaging, Aalto University. During acquisition, the data were filtered to $0.1-330 \mathrm{~Hz}$ and sampled at $1 \mathrm{kHz}$. Prior to the MEG recording, anatomical landmarks (nasion, left and right preauricular points), head-position indicator coils, and additional scalp-surface points (around 100) were digitized using an Isotrak 3D digitizer (Polhemus Navigational Sciences, Colchester, VT, USA). Bipolar electrooculogram (EOG) with electrodes positioned around the right eye (laterally and below) was recorded. Fourteen of the 15 subjects were recorded with continuous head movement tracking. All subjects were measured in the seated position. The back-projection screen was $1 \mathrm{~m}$ from the eyes of the subject. If needed, vision was corrected by nonmagnetic goggles.

\subsection{Data pre-processing}

The MaxFilter software (version 2.2.10; Elekta Oy/MEGIN, Helsinki, Finland) was applied to suppress external interference using temporal signal space separation to compensate for head movements (Taulu and Hari, 2009). Further analysis was performed using MNE version 2.7.4 and MNE-Python (version 0.14; Gramfort et al., 2014) and ScikitLearn (version 0.18; Pedregosa et al., 2011) software packages.

Finite-impulse-response (FIR) filters were employed to filter the unaveraged MEG data to $0.1-30 \mathrm{~Hz}$ for visualization of the evoked responses and for sensor- and source-level decoding. Ocular artifacts were suppressed by removing those independent components (1-4 per subject, on average 3 ) that correlated most with the EOG signal. 2-s long epochs with a 0.50 -s prestimulus period were extracted from the data at every word stimulus. The delay in the sound reproduction system was considered in the epoch timing. Epochs were rejected if any of the gradiometer signals exceeded $4000 \mathrm{fT} / \mathrm{cm}$. Responses to deviants were excluded from data analysis.

\subsection{Evoked responses}

\subsubsection{Sensor-level analysis}

The trial counts were equalized across the conditions ("Attended Left", "Attended Right", "Unattended Left" and "Unattended Right") and the trials were averaged. Only attended attention conditions were used in sensorand source-level classification. 


\subsubsection{Source-level analysis}

Head models were constructed based on individual magnetic resonance images (MRIs) when available $(N=12)$ applying the watershed algorithm implemented in the FreeSurfer software (Version 5.3; Dale et al., 1999; Fischl et al., 1999). Using the MNE software, single-compartment boundary element models (BEM) comprising 5120 triangles were then created based on the inner skull surface. The MRIs of three subjects were not available and these subjects were excluded from the source-level analysis.

For the source space, the cortical mantle was segmented from MRIs using FreeSurfer and the resulting triangle mesh was subdivided to 4098 sources per hemisphere. The dynamic statistical parametric mapping (dSPM; Dale et al., 2000) variant of minimum-norm estimation was applied to model the activity at these sources. The noise covariance used in the model was estimated for each subject from all epochs' 0.50 -s pre-stimulus intervals. dSPM sources "Attended Left" and "Attended Right" attention conditions were estimated for all subjects individually. The obtained source amplitudes were then normalized for each subject and a group-level dSPM source estimate was calculated by morphing the normalized individual estimates to the FreeSurfer average brain and averaging them. For the group averages individual dSPMs were normalized by putting source peak value to 1 .

\subsection{Classification}

\subsubsection{Sensor-level classification}

A linear support vector machine (SVM; Cortes and Vapnik, 1995) implemented in the ScikitLearn package (Pedregosa et al., 2011) was applied for single-epoch classification of the conditions "Attended Left" vs. "Attended Right". To this end, the pre-processed MEG data (filtered to 0.1-30 Hz) were down-sampled by factor 8 to a sampling rate of $125 \mathrm{~Hz}$. Amplitudes of the planar gradiometer channels were concatenated to form the feature vector. Five-fold cross-validation (CV) was applied with an 80/20 split; $80 \%$ of data were used for training and the rest for testing. The empirical chance level was around $55 \%$ for our sample size of 500 trials in this two-class decoding task (Combrisson and Jerbi, 2015).

Decoding was separately performed on data of 1$)$ the entire epoch (250 samples x 204 channels; entire-epoch decoding), 2) one time point (1 sample x 204 channels; spatially-resolved decoding), and 3) one channel (250 samples x 1 channel; time-resolved decoding). 


\subsubsection{Source-level classification}

A linear SVM decoder with five-fold CV (80/20 split) was applied to the individual source estimates calculated for the conditions "Attended Left" and "Attended Right". A spatial searchlight across the source space was used on the 2-s epochs and the resulting accuracy maps were morphed to the FreeSurfer average brain (comprising 20484 source points) and averaged. In addition, the accuracies obtained for the left and right auditory cortex were compared across the individuals using a paired t-test. 


\section{Results}

\subsection{Behavioral data}

The average relative absolute error of the reported deviant count was $49 \%$ for the $10-\%$ deviant probability $(N=5$; subjects S03-S07) and $12 \%$ for the $5-\%$ probability $(N=7$; subjects $\mathrm{S} 09-\mathrm{S} 15)$.

\begin{tabular}{c|c} 
Subject & Score $($ mean \pm SD; \%) \\
\hline S01 & $90 \pm 1$ \\
S02 & $95 \pm 2$ \\
S03 & $97 \pm 1$ \\
S04 & $92 \pm 1$ \\
S05 & $99 \pm 1$ \\
S06 & $84 \pm 2$ \\
S07 & $87 \pm 2$ \\
S08 & $96 \pm 0$ \\
S09 & $91 \pm 3$ \\
S10 & $94 \pm 1$ \\
S11 & $96 \pm 1$ \\
S12 & $97 \pm 1$ \\
S13 & $90 \pm 1$ \\
S14 & $99 \pm 1$ \\
S15 & $84 \pm 2$ \\
\hline MEAN & $93 \pm 1$ \\
\hline
\end{tabular}

Table 1: Entire-epoch classification accuracy for all subjects and the group mean accuracy.

\subsection{Sensor-level analysis}

Average evoked responses to each attention condition ("Attended Left", "Unattended Left", "Attended Right", "Unattended Right") are shown in Figures 2 and 3.

Time-resolved classification revealed that the most informative responses occurred in 100-400 ms after the stimulus onset (Figure 2 and 3). The average evoked responses for subject S03 (group) peaked at $185 \mathrm{~ms}$ (185 $\mathrm{ms}), 236 \mathrm{~ms}(245 \mathrm{~ms})$ and $309 \mathrm{~ms}(312 \mathrm{~ms})$ for "Attended Left" attention condition. For the condition "Attended Right", responses peaked at $193 \mathrm{~ms}$ (195 ms), $273 \mathrm{~ms}$ (304 ms) and $361 \mathrm{~ms}$ (390 ms). Both in Subject S03 and in the group, time-resolved classification peaked at $320 \mathrm{~ms}$. 
A

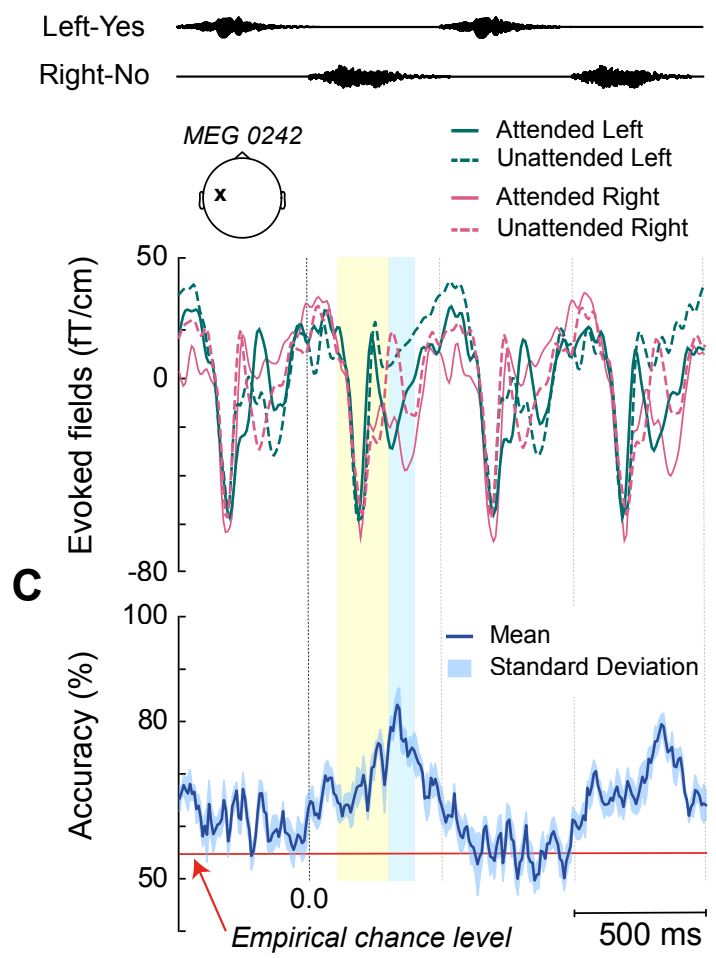

B

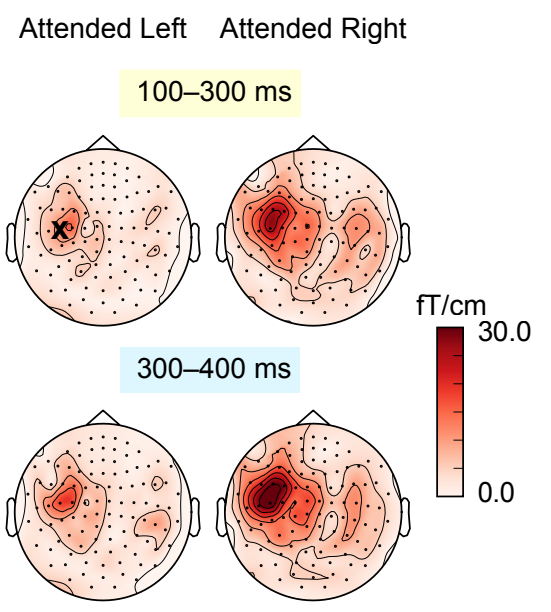

D

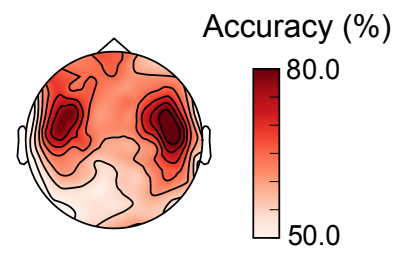

Figure 2: Evoked responses and classification accuracies in a representative subject (S03) to four consecutive stimuli. A: Acoustic waveforms of the stimuli and average responses at a planar gradiometer channel low-pass-filtered at $30 \mathrm{~Hz}$ in the attended (solid lines) and unattended (dashes lines) conditions. B: Spatial patterns (gradient strength maps) of the evoked responses. C: Time-resolved classification accuracies with standard deviation (blue shading) computed across the folds. D: Spatially-resolved classification accuracies. 
A

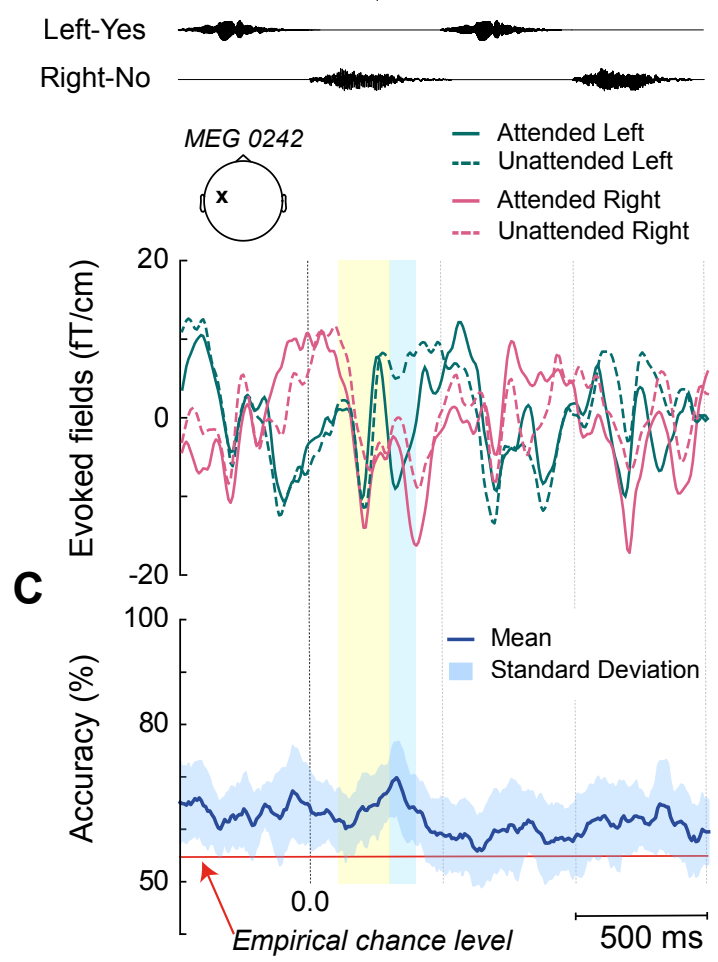

B

\section{Attended Left Attended Right}

$100-300 \mathrm{~ms}$

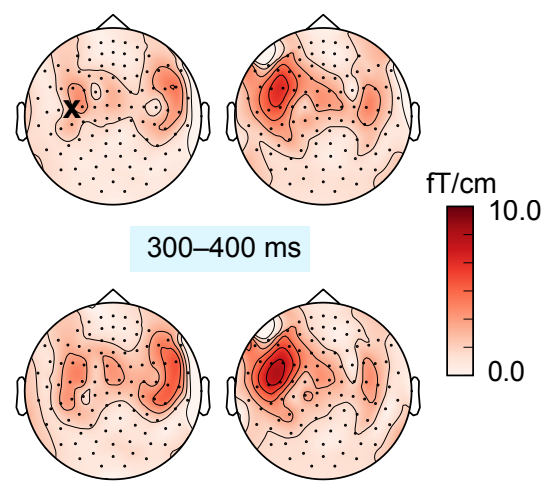

D

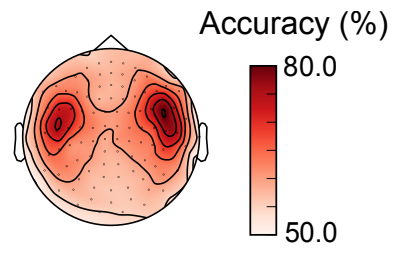

Figure 3: Evoked responses and classification accuracies at the group level $(N=15)$. A: Acoustic waveforms of the stimuli and average responses at a planar gradiometer channel low-pass-filtered at $30 \mathrm{~Hz}$ in the attended (solid lines) and unattended (dashes lines) conditions. B: Spatial patterns (gradient strength maps) of the evoked responses. C: Time-resolved classification accuracies with standard deviation (blue shading) computed across the folds. D: Spatially-resolved classification accuracies. 
bioRxiv preprint doi: https://doi.org/10.1101/588491; this version posted March 27, 2019. The copyright holder for this preprint (which was not certified by peer review) is the author/funder, who has granted bioRxiv a license to display the preprint in perpetuity. It is made available under aCC-BY-NC-ND 4.0 International license.

\section{Attention conditions}

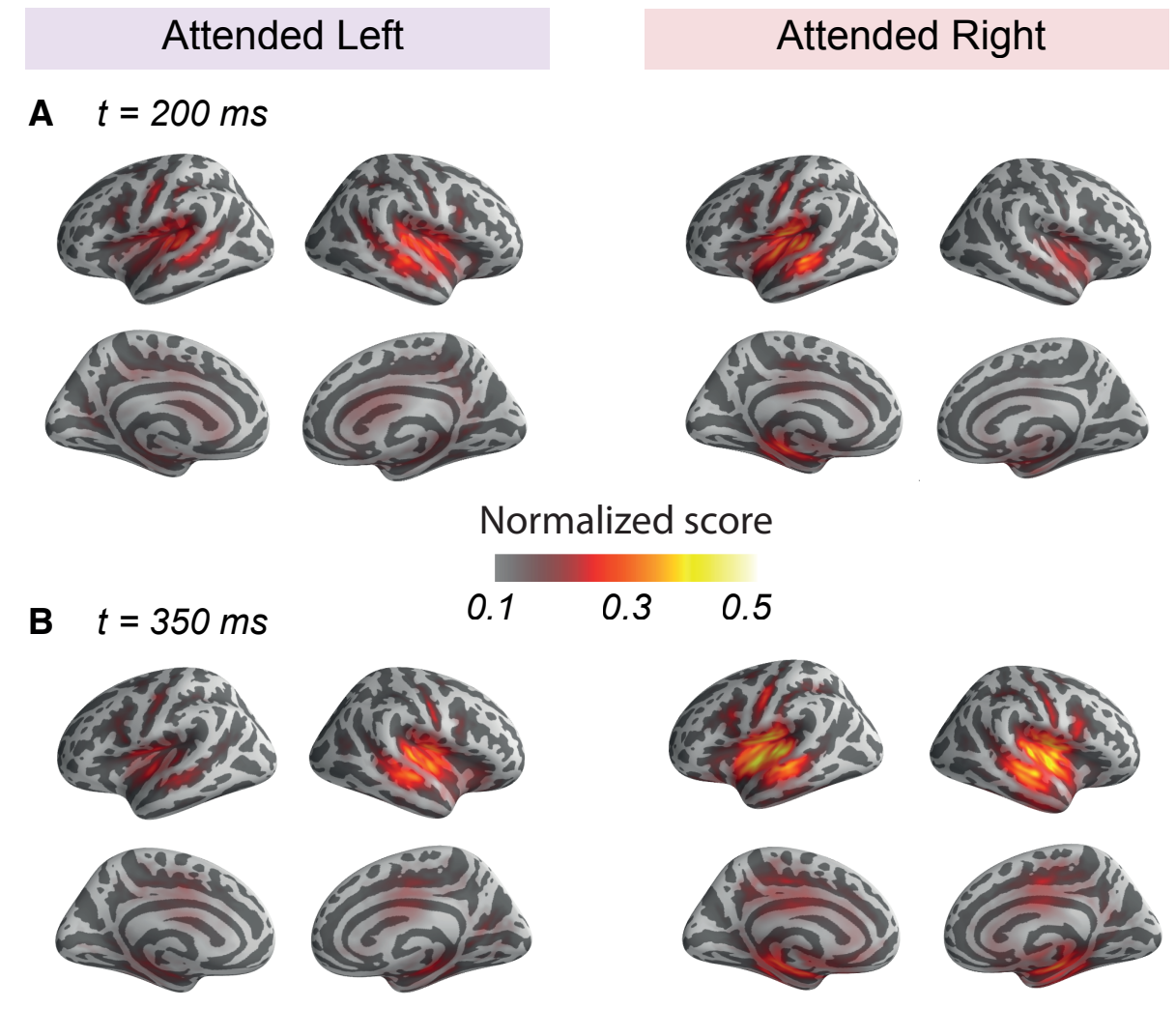

Figure 4: Source estimates of the evoked responses. Normalized group average $(N=12)$. A: "Attended Left" and "Attended Right" at $200 \mathrm{~ms}$ after stimulus onset; B: "Attended Left" and "Attended Right" at 350 ms after stimulus onset . 


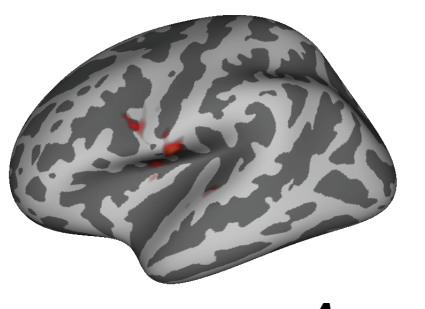

Accuracy, \%

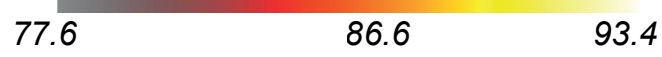

Figure 5: Source-level spatial-searchlight classification in a representative subject (S03) morphed to the average brain. Color indicates the accuracy of decoding "Attended Left" vs. "Attended Right" based on the signal from that cortical location; the top $5 \%$ of the scores in each hemisphere are shown.

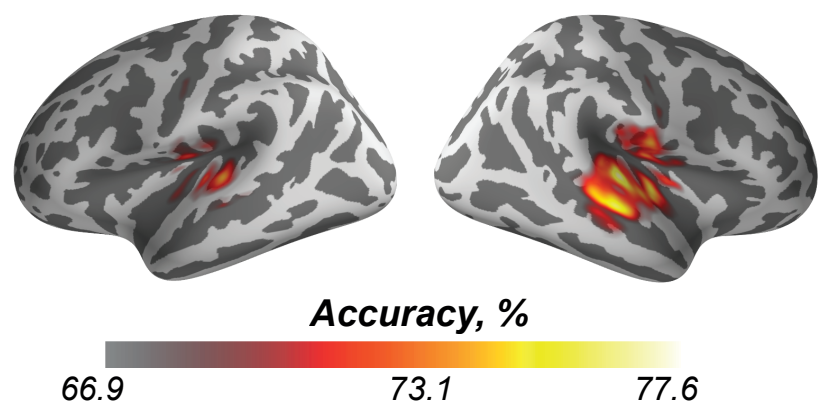

Figure 6: Source-level decoding at the group level $(N=12)$. Color indicates the accuracy of classification of "Attended Left" vs. "Attended Right" based on the signal from that cortical location; the top $5 \%$ of the scores in each hemisphere are shown. 
Spatially-resolved classification indicated that the most informative signals arose from temporal regions. Both the temporal and spatial decoding patterns were qualitatively similar across the subjects; see Figure 2 for a representative subject and Figure 3 for the group result. Using the entire epochs for decoding "Attend Left" vs. "Attend Right" conditions yielded scores 84-99\% (mean 93\%; Table 1) across the 15 subjects.

\subsection{Source-level analysis}

Source modeling of the peaks of the evoked responses indicated sources in both auditory cortices. Paired t-tests showed significant source-amplitude differences $(p<0.05)$ between the left and right hemisphere sources in the "Attended Left" condition but not in the "Attended Right" condition $(p>$ $0.05 ; N=12$ ). In addition, a paired t-test showed that in the "Attended Left" condition, the source amplitudes were significantly different from those in the "Attended Right" condition at $350 \mathrm{~ms}(p<0.05)$ (see Figure 6) while at $200 \mathrm{~ms}$ the difference was not significant.

Spatial-searchlight decoding applied in source space indicated that auditory cortices were the most informative about attention target; see Fig. 6 . All subjects ( $N=12$ with source estimates) showed the highest accuracy for source signals arising from the auditory cortices. The across-subjects average peak value was $74.0 \%$ in the left and $77.6 \%$ in the right temporal areas. This difference between the hemispheres was not significant $(p=0.389, N=12$ ). 


\section{Discussion}

In this paper, we showed that the target of selective auditory attention to concurrent streams of naturalistic speech stimuli can be robustly detected from unaveraged MEG responses and that this detection is most accurate for signals arising from the auditory cortices 300-400 ms after stimulus onset.

In our data, the earliest clearly-discernible response peaks at about $200 \mathrm{~ms}$ after the onset of the spoken-word stimulus. This response - often referred to as N2 or N200 in EEG literature - shows only weak dependence on attention in our results. In contrast, the responses occurring within 300-400 ms are significantly modulated by attention. Several studies have shown that the late component of the P300 response is affected by attention (see e.g. Chennu et al., 2013; Picton, 1992) and this component is likely the largest contributor to our classification results.

In general, an increased P300 amplitude can be due to unexpected changes in the stimulus sequence (e.g. in an auditory oddball task). As opposed to the mismatch negativity (MMN) response occurring earlier and indexing local deviants (Näätänen et al., 2007), the P300 appears to reflect mostly global, consciously-perceived changes in the stimulus stream, e.g. an unexpected stimulus sequence (Bekinschtein et al., 2009). These observations provide further evidence that the P300 response echoes cognitive processes, such as attention, that are closely linked to conscious perception.

To elicit brain responses with maximal attentional modulation but with minimal subject training, we employed meaningful stimuli that are easy to attend to even during dichotic listening. As pointed out by Hill and colleagues (2014), applying naturalistic stimuli as opposed to meaningless tone pips could make dichotic listening more pleasant and thus contribute to stronger attentional modulation of the responses and eventually to higher accuracy in classifying the target of attention.

Due to the above factors and the obtained high classification accuracy, our paradigm could be well-suited for a brain-computer interface (BCI). Several studies have exploited selective auditory attention and/or P300 responses to drive a BCI but usually not with real spoken words. For example, Halder and colleagues (2018) used five Japanese Hiragana syllables $(/ k a /, / k i /, / k u /, / k e /$, and $/ k o /)$ presented at different spatial locations in the auditory scene while measuring EEG, applied shrinkage Linear Discriminant Analysis (LDA) to classify the target of attention from the P300 responses, and obtained classification accuracy of about 70\%. Sugi and col- 
leagues (2018) similarly employed spatially distinct sound sources (six in their case) and optimized the stimulus onset asynchrony for maximal information transfer rate; the optimal SOA was found to be 400-500 ms, which yielded over $85 \%$ accuracy when classifying the target sound source vs. all others. Heo and colleagues (2017) utilized piano and violin music, sounds of nature as well as pure tones which were all amplitude modulated at 38 and $42 \mathrm{~Hz}$ to elicit auditory steady-state responses. LDA classification of the EEG responses to sounds of nature yielded the highest accuracy (83\%), and the authors argue that this due to the acceptance, or pleasantness, of these stimuli compared to the other stimuli in that study.

The high classification accuracy we have now obtained offline does not readily indicate high online accuracy. In an online setting, the classifier can only be trained with samples from the beginning of the recording, which may lower the classification accuracy if the responses evolve in the course of the measurement session due to adaptation or change in the mental strategy to maintain attention in one stream. In addition, all the pre-processing that we now perform offline to improve data quality may not be available online due to computational reasons.

Individual differences in response latencies and spatial patterns on the MEG sensor array may limit across-subject generalization of trained classifiers. Future studies could assess these differences and their impact on classification accuracy.

Our current results are based on MEG measurements. As a non-portable and expensive technology, MEG-based BCIs have limited applications beyond neuroscientific experimentation. However, a MEG BCI could assist the development of an eventual EEG-based BCI that could be adopted widely.

Despite the current limitations above, our paradigm and classification approach holds promise for a future BCI. The use of stimuli that directly carry the semantics of the communication or control elements and an intuitive selection task make such a BCI easy to use and likely reduce the training time of both the subject and the classifier. 


\section{Conclusions}

We have shown that the target of auditory attention to one of two concurrent streams spoken words can be robustly decoded from single MEG responses. Our result corroborates attentional modulation of auditory evoked responses also to naturalistic stimuli. The achieved high decoding accuracy could enable the use of our experimental paradigm and classification method in an efficient and intuitive brain-computer interface.

\section{Acknowledgements}

The measurements were conducted at the MEG Core of Aalto Neuroimaging, Aalto University, Finland. Measurements were financially supported by Aalto Brain Centre. Authors declare no conflicts of interest.

\section{References}

Astrand, E., Wardak, C., Ben Hamed, S., 2014. Selective visual attention to drive cognitive brain-machine interfaces: from concepts to neurofeedback and rehabilitation applications. Front. Syst. Neurosci. 8, 144. doi:10. 3389/fnsys . 2014.00144.

Bekinschtein, T.A., Dehaene, S., Rohaut, B., Tadel, F., Cohen, L., Naccache, L., 2009. Neural signature of the conscious processing of auditory regularities. Proc. Natl. Acad. Sci. U S A 106, 1672-1677. doi:10.1073/pnas. 0809667106.

Chennu, S., Noreika, V., Gueorguiev, D., Blenkmann, A., Kochen, S., Ibáñez, A., Owen, A.M., Bekinschtein, T.A., 2013. Expectation and attention in hierarchical auditory prediction. J. Neurosci. 33, 11194-11205. doi:10. 1523/JNEUROSCI . 0114-13.2013.

Cherry, E.C., 1953. Some experiments on the recognition of speech, with one and with two ears. J. Acoust. Soc. Am. 25, 975-979. doi:10.1121/1. 1907229.

Combrisson, E., Jerbi, K., 2015. Exceeding chance level by chance: The caveat of theoretical chance levels in brain signal classification and statistical assessment of decoding accuracy. J. Neurosci. Methods 250, 126-136. doi:10.1016/j.jneumeth.2015.01.010. 
Cortes, C., Vapnik, V., 1995. Support-vector networks. Mach. Learn. 20, 273-297. doi:10.1023/A : 1022627411411.

Curtin, A., Ayaz, H., Liu, Y., Shewokis, P.A., Onaral, B., 2012. A p300-based EEG-BCI for spatial navigation control. Conf. Proc. IEEE Eng. Med. Biol. Soc. 2012, 3841-3844. doi:10.1109/EMBC. 2012.6346805.

Dale, A.M., Fischl, B., Sereno, M.I., 1999. Cortical surface-based analysis. i. segmentation and surface reconstruction. NeuroImage 9, 179-194. doi:10. 1006/nimg. 1998.0395.

Dale, A.M., Liu, A.K., Fischl, B.R., Buckner, R.L., Belliveau, J.W., Lewine, J.D., Halgren, E., 2000. Dynamic statistical parametric mapping: combining fMRI and MEG for high-resolution imaging of cortical activity. Neuron 26, 55-67. doi:10.1016/S0896-6273(00)81138-1.

Fischl, B., Sereno, M.I., Dale, A.M., 1999. Cortical surface-based analysis. II: Inflation, flattening, and a surface-based coordinate system. NeuroImage 9, 195-207. doi:10.1006/nimg.1998.0396.

Furdea, A., Halder, S., Krusienski, D.J., Bross, D., Nijboer, F., Birbaumer, N., Kübler, A., 2009. An auditory oddball (p300) spelling system for brain-computer interfaces. Psychophysiology 46, 617-625. doi:10.1111/ j.1469-8986.2008.00783.x.

Geronimo, A., Kamrunnahar, M., Schiff, S.J., . Single trial predictors for gating motor-imagery brain-computer interfaces based on sensorimotor rhythm and visual evoked potentials. Front. Neurosci. 10. doi:10.3389/ fnins.2016.00164.

Gramfort, A., Luessi, M., Larson, E., Engemann, D.A., Strohmeier, D., Brodbeck, C., Parkkonen, L., Hämäläinen, M.S., 2014. Mne software for processing meg and eeg data. NeuroImage 86, $446-460$.

Halder, S., Rea, M., Andreoni, R., Nijboer, F., Hammer, E.M., Kleih, S.C., Birbaumer, N., Kübler, A., 2010. An auditory oddball brain-computer interface for binary choices. Clin. Neurophysiol. 121, 516-523. doi:10. 1016/j.clinph.2009.11.087. 
Halder, S., Takano, K., Kansaku, K., 2018. Comparison of four control methods for a five-choice assistive technology. Front. Hum. Neurosci. 12, 228. doi:10.3389/fnhum. 2018.00228.

Halder, S., Takano, K., Ora, H., Onishi, A., Utsumi, K., Kansaku, K., 2016. An evaluation of training with an auditory p300 brain-computer interface for the japanese hiragana syllabary. Front. Neurosci. 10, 446. doi:10. 3389/ fnins.2016.00446.

Heo, J., Baek, H.J., Hong, S., Chang, M.H., Lee, J.S., Park, K.S., 2017. Music and natural sounds in an auditory steady-state response based braincomputer interface to increase user acceptance. Comput. Biol. Med. 84, 45-52. doi:10.1016/j . compbiomed.2017.03.011.

Hill, N.J., Moinuddin, A., Häuser, A.K., Kienzle, S., Schalk, G., 2012. Communication and control by listening: toward optimal design of a two-class auditory streaming brain-computer interface. Front. Neurosci. 6, 181. doi:10.3389/fnins. 2012.00181.

Hill, N.J., Ricci, E., Haider, S., McCane, L.M., Heckman, S., Wolpaw, J.R., Vaughan, T.M., 2014. A practical, intuitive brain-computer interface for communicating 'yes' or 'no' by listening. J. Neural. Eng. 11, 035003. doi:10 . 1088/1741-2560/11/3/035003.

Hillyard, S.A., Hink, R.F., Schwent, V.L., Picton, T.W., 1973. Electrical signs of selective attention in the human brain. Science 182, 177-180. doi:10.1126/science.182.4108.177.

Höhne, J., Schreuder, M., Blankertz, B., Tangermann, M., 2011. A novel 9-class auditory ERP paradigm driving a predictive text entry system. Front. Neurosci. 5, 99. doi:10.3389/fnins. 2011.00099.

Hübner, D., Schall, A., Prange, N., Tangermann, M., 2018. Eyes-closed increases the usability of brain-computer interfaces based on auditory eventrelated potentials. Front. Hum. Neurosci. 12, 391. doi:10.3389/fnhum. 2018.00391.

Kaongoen, N., Jo, S., 2017. A novel hybrid auditory BCI paradigm combining ASSR and p300. J. Neurosci. Methods 279, 44-51. doi:10.1016/j. jneumeth.2017.01.011. 
Kidd, G.J., 2017. Enhancing auditory selective attention using a visually guided hearing aid. J. Speech Lang. Hear. Res. 60, 3027-3038. doi:10 . 1044/2017_JSLHR-H-17-0071.

Kim, D.W., Hwang, H.J., Lim, J.H., Lee, Y.H., Jung, K.Y., Im, C.H., 2011. Classification of selective attention to auditory stimuli: toward vision-free brain-computer interfacing. J. Neurosci. Methods 197, 180-185. doi:10 . $1016 / j \cdot$ jneumeth. 2011.02.007.

McCane, L.M., Heckman, S.M., McFarland, D.J., Townsend, G., Mak, J.N., Sellers, E.W., Zeitlin, D., Tenteromano, L.M., Wolpaw, J.R., Vaughan, T.M., 2015. P300-based brain-computer interface (BCI) event-related potentials (ERPs): People with amyotrophic lateral sclerosis (ALS) vs. agematched controls. Clin. Neurophysiol. 126, 2124-2131. doi:10.1016/j . clinph.2015.01.013.

Nambu, I., Ebisawa, M., Kogure, M., Yano, S., Hokari, H., Wada, Y., 2013. Estimating the intended sound direction of the user: Toward an auditory brain-computer interface using out-of-head sound localization. PLoS ONE 8, e57174. doi:10.1371/journal.pone.0057174.

Nijboer, F., Furdea, A., Gunst, I., Mellinger, J., McFarland, D.J., Birbaumer, N., Kübler, A., 2008. An auditory brain-computer interface (BCI). J. Neurosci. Methods 167, 43-50. doi:10.1016/j.jneumeth.2007.02.009.

Näätänen, R., Paavilainen, P., Rinne, T., Alho, K., 2007. The mismatch negativity $(\mathrm{MMN})$ in basic research of central auditory processing: a review. Clin. Neurophysiol. 118, 2544-2590. doi:10.1016/j . clinph. 2007. 04.026 .

Pedregosa, F., Varoquaux, G., Gramfort, A., Michel, V., Thirion, B., Grisel, O., Blondel, M., Prettenhofer, P., Weiss, R., Dubourg, V., Vanderplas, J., Passos, A., Cournapeau, D., Brucher, M., Perrot, M., Duchesnay, É., 2011. Scikit-learn: Machine learning in python. J. Mach. Learn. Res. 12, 2825-2830.

Peirce, J.W., 2007. PsychoPy-psychophysics software in python. J. Neurosci. Methods 162, 8-13. doi:10.1016/j.jneumeth.2006.11.017.

Peirce, J.W., 2008. Generating stimuli for neuroscience using PsychoPy. Front. Neuroinform. 2, 10. doi:10.3389/neuro.11.010.2008. 
Picton, T.W., 1992. The p300 wave of the human eventrelated potential. Clin. Neurophysiol. 9, 456-479. doi:10.1097/ 00004691-199210000-00002.

Schreuder, M., Blankertz, B., Tangermann, M., 2010. A new auditory multiclass brain-computer interface paradigm: Spatial hearing as an informative cue. PLoS ONE 5, e9813. doi:10.1371/journal.pone.0009813.

Sellers, E.W., Donchin, E., 2006. A p300-based brain-computer interface: Initial tests by ALS patients. Clin. Neurophysiol. 117, 538-548. doi:10 . 1016/j.clinph.2005.06.027.

Sugi, M., Hagimoto, Y., Nambu, I., Gonzalez, A., Takei, Y., Yano, S., Hokari, H., Wada, Y., 2018. Improving the performance of an auditory braincomputer interface using virtual sound sources by shortening stimulus onset asynchrony. Front. Neurosci. 12, 108. doi:10.3389/fnins. 2018.00108.

Taulu, S., Hari, R., 2009. Removal of magnetoencephalographic artifacts with temporal signal-space separation: demonstration with single-trial auditory-evoked responses. Hum. Brain Mapp. 30, 1524-1534. doi:10 . 1002/hbm. 20627.

Woldorff, M., Gallen, C., Hampson, S., Hillyard, S., Pantev, C., Sobel, D., Bloom, F., 1993. Modulation of early sensory processing in human auditory cortex during auditory selective attention. Proc. Natl. Acad. Sci. U S A 90, 8722-8726. doi:10.1073/pnas.90.18.8722.

Yeom, S.K., Fazli, S., Müller, K.R., Lee, S.W., 2014. An efficient ERPbased brain-computer interface using random set presentation and face familiarity. PLoS ONE 9, e111157. doi:10.1371/journal .pone.0111157. 